\title{
Retrospective study of prognostic importance of DNA flow cytometry of urinary bladder carcinoma
}

\author{
C E M BLOMJOUS, N W SCHIPPER, J P A BAAK, E M VAN GALEN, \\ H J DE VOOGT, * C J L M MEYER
}

From the Department of Pathology and the *Department of Urology, Free University Hospital, Amsterdam, The Netherlands

SUMMARY Cellular DNA content was determined by flow cytometry on routinely processed paraffin sections of 61 primary and untreated transitional cell carcinomas of the urinary bladder, and correlated with tumour grade and stage and clinical follow up. All 16 $(25 \%)$ grade 1 carcinomas were diploid and all $11(20 \%)$ grade 3 tumours were aneuploid. The $34(55 \%)$ grade 2 carcinomas comprised $13(40 \%)$ diploid and $21(60 \%)$ aneuploid cases. Among the 37 superficial carcinomas (stage Ta and T1), 25 (65\%) were diploid; 20 (85\%) of the 24 advanced tumours (stage T2 to T4) had aneuploid tracings. Ploidy was a significant prognostic indicator (p: 0.006) of five year survival. The initial presence of aneuploidy in superficial bladder carcinoma (stage Ta and T1) is a strong argument for more aggressive treatment than is customary.

The most important prognostic variables of transitional cell carcinoma of the urinary bladder to date are tumour grade and stage, ${ }^{1}$ but additional criteria are needed if treatment is to be more effective. Several histological grading systems are used, ${ }^{2-4}$ which are all based on estimation of the degree of histological and cytonuclear atypia: this carries a risk of observer variation. ${ }^{56}$ Furthermore, although survival depends on histological grade, ${ }^{23}$ grade 2 tumours in particular (World Health Organisation classification, 1973) form a heterogeneous group, and patient survival varies enormously. Tumour stage does not always provide sufficient prognostic information either, particularly among patients with superficial tumours (stage $\mathrm{Ta}$ and $\mathrm{T} 1$ ). These patients are usually conservatively treated, and a large proportion will experience recurrence and 15 to $25 \%$ will ultimately suffer progressive recurrence- that is, high grade tumour or invasive disease. ${ }^{78}$

Examples of more objective predictors of clinical behaviour are cytogenetic and cytophotometric characteristics, the pattern of immunohistological markers, such as carcinoembryonic antigen, $\mathrm{ABO}(\mathrm{H})$ and $T$ antigen, and ultrastructural changes such as changes in the cell membrane and attachment sites. ${ }^{9-19}$ Although promising, requirement for technologically advanced techniques or lack of

Accepted for publication 18 June 1987 reproducibility have ensured that so far none of them has emerged as suitable for routine practice.

In recent years flow cytometry has been developed as a fast and objective method of analysing nuclear DNA in large numbers of cells. In urology this technique has been mainly used for scanning cytological specimens to monitor patients with conservatively managed carcinoma. ${ }^{19-23}$ Flow cytometry has shown good correlation of ploidy with tumour grade and stage, ${ }^{2024}$ but only a few studies have evaluated its impact on clinical outcome. The recent development of a method for flow cytometric analysis of routinely processed paraffin embedded histological material has opened the laboratory archives for retrospective analysis of patients whose clinical follow up has been documented. ${ }^{25}$

In this study we investigated whether flow cytometry on paraffin embedded tissue of the initial bladder tumour yielded prognostic information in addition to that provided by tumour grade and stage, with special reference to grade 2 carcinoma and to non-invasive tumours (stage $\mathrm{Ta}$ and $\mathrm{T} 1$ ).

\section{Material and methods}

Of 121 patients who had undergone transurethral resection of a primary and untreated transitional cell carcinoma of the urinary bladder between September 1974 and August 1979, 61 were included in this study. Criteria were that: archival paraffin blocks still were 
available; tumour specimens had been properly fixed in $5 \%$ neutral buffered formalin; and cauterisation artefacts were not abundant. There were no differences in age, sex, and tumour stage between the original and selected group.

One representative tissue block was chosen from each specimen and stained with hematoxylin and eosin: stained sections were examined independently by two pathologists. Tumour grade was established according to the World Health Organisation classification. ${ }^{4}$ Gradings given by both pathologists were considered in addition to the grade originally arrived at by routine diagnostic procedures. Complete agreement between the observations made by the two pathologists and the WHO classification was obtained for $65 \%$ of the cases and no differences of more than one grade occurred. In cases of disagreement the prevailing grade was assessed. Sixteen cases were classified as grade 1 carcinoma, 34 as grade 2, and 11 as grade 3 .

The tumours were also staged in accordance with the tumours, nodes, metastases (TNM) classification. ${ }^{26}$ Thirty seven patients had superficial carcinoma (stage $\mathrm{Ta}$ and $\mathrm{T} 1$ ) and 24 patients had advanced disease (stage T2 to T4) (table 1).

\section{CLINICAL FEATURES}

Clinical data were available from 58 patients; three patients were lost to follow up. Forty four patients were male and 14 were female; age ranged from 46 to 88 years, mean 68.9 years. All patients with superficial carcinoma (stage $\mathrm{Ta}$ and $\mathrm{T} 1$ ) had been initially treated by transurethral section alone. Patients with advanced disease (stage T2 T4), depending on general health condition and likelihood of cure, were given different modes of treatment-local radium implantation (three of 22), radical cystectomy with adjuvant radiotherapy (seven of 22), and conservative radiotherapy (10 of 22), or they remained untreated (two of 22).

\section{FLOW CYTOMETRY}

Cellular DNA in paraffin embedded specimens was analysed using the method described by Hedley $e t$ $a l,{ }^{25}$ with slight modifications. Sections $(50 \mu \mathrm{m})$ were cut from selected tissue blocks and the sections de-

Table 1 Distribution of tumour grade and stage

\begin{tabular}{lrrrrrrl}
\hline & \multicolumn{3}{c}{ Stage } & & & \\
\cline { 2 - 6 } & $T a$ & $T I$ & $T 2$ & $T 3$ & $T 4$ & Total \\
\hline Grade 1 & 14 & 2 & 0 & 0 & 0 & 16 \\
Grade 2 & 7 & 13 & 8 & 1 & 5 & 34 \\
Grade 3 & 0 & 1 & 6 & 1 & 3 & 11 \\
Total & 21 & 16 & 14 & 2 & 8 & 61 \\
\hline
\end{tabular}

waxed in xylol, rehydrated, and enzymatically dispersed by protease (Sigma P-5255, 0.05\% in 0.9\% buffered saline, $\mathrm{pH}=7$ ), vigorously vortex mixed. After filtration through a $50 \mu \mathrm{m}$ nylon gauze centrifugation (for five minutes at $1000 \mathrm{rpm}$ ), resuspension in carbowax ( $2 \%$ polyethylene glycol in $50 \%$ ethanol), and mechanical detachment by repeated syringing (through 21 gauge needles), the cell suspension was centrifuged, washed in Tris-hydrochloric acid buffer, and recentrifuged. The cells then were stained with the DNA-fluorochrome 4',6'-diaminido-2-phenylindole dihydrochloride (final concentration $2 \mu \mathrm{q}$ DAPI/100 ml Tris-hydrochloric acid buffer).

Cellular DNA was analysed with the PAS II flow cytometer (Partec, Arlesheim, Switzerland), using excitation light at $350 \mathrm{~nm}$. Suspensions of mouse thymocytes were used for instrument setting. For each histogram 30000 to 80000 cells were scanned. The first modal cell peak was regarded as the diploid peak. Samples were considered to be aneuploid when in addition to the $G_{0} / G_{1}$ and $G_{2} / M$ peaks, one or more DNA peaks were detected. Tumours in which the proportion of peritetraploid cells (DNA indices between 1.9 and $2 \cdot 1$ ) exceeded $10 \%$ of the whole cell population were regarded as peritetraploid.

Significance of ploidy in relation to tumour grade and stage was determined by the $\chi^{2}$ test. Survival (Kaplan-Meier) curves were analysed, using the Mantel-Cox statistics. The $\chi^{2}$ test was used to evaluate the relation between ploidy and recurrence and progression. A $p$ value of $<0.05$ was regarded as significant.

\section{Results}

\section{FLOW CYTOMETRY}

Flow cytometry of all 61 specimens yielded DNA histograms from which could be determined ploidy, although background activity by cell debris resulted in an overall mean coefficient of variation of the $G_{0} / G_{1}$ peak of 6.51 (SD 2.33). Although the storage period of paraffin tissue blocks varied from seven to 12 years, the coefficient of variation had not been notably influenced and some of the oldest specimens had relatively low coefficients of variation. As a high coefficient of variation, which corresponds to a wide $\mathrm{G}_{0} / \mathrm{G}_{1}$ peak, carries with it some risk of overlooking nearby diploid aneuploid populations, the diploid cases with a coefficient of variation of less than 5.5 were compared with those more than 5.5. Both subgroups did not exhibit any difference in distribution of tumour grade and stage or clinical outcome. Thus, although some of the wide diploid peaks might have resulted from an unrecognised nearly diploid peak, these tumours did not need to be divided into subgroups. DNA histograms of 29 tumours gave diploid 
Table 2 Ploidy in grades 1-3 tumours

\begin{tabular}{lcccc}
\hline & & \multicolumn{2}{l}{ Aneuploid } & \\
\cline { 3 - 5 } & Diploid & Peritetraploid & Non-peritetraploid & Total \\
\hline Grade 1 & 16 & 0 & 0 & 16 \\
Grade 2 & 13 & 12 & $9^{*}$ & 34 \\
Grade 3 & 0 & 1 & $10 \dagger$ & 11 \\
Total & 29 & 13 & 19 & 61 \\
\hline
\end{tabular}

*Including one multiploid tumour; tincluding three multiploid tumours.

cell lines; the remaining 32 cases were aneuploid (including 13 peritetraploid tumours and four with multiple aneuploid peaks).

PLOIDY IN RELATION TO HISTOLOGICAL GRADE Ploidy was strongly related to histological grade. All grade 1 carcinomas were diploid and all grade 3 tumours were aneuploid. Grade 2 carcinomas comprised 13 diploid $(40 \%)$ and 21 aneuploid cases $(60 \%)$ (table 2). More than half of the aneuploid grade 2 lesions (12 of 21) showed peritetraploidy; grade 3 tumours showed non-peritetraploid aneuploid tracings (10 of 11) $(\mathrm{p}=0.009)$. Of the four multiploid tumours, 3 were grade 3 and 1 grade 2 .

\section{PLOIDY IN RELATION TO STAGE OF DISEASE}

There was a strong correlation between ploidy and tumour stage. Distribution of ploidy and stage is shown in table 3. Of the superficial tumours (stage Ta and $\mathrm{T} 1$ ), $35 \%$ were aneuploid (12 of 37 ), and most (seven of 12) fell into the peritetraploid category. Only $15 \%$ (three of 21 ) of the non-invasive tumours (stage Ta) were aneuploid; more than half (nine of 16) of the lesions with invasion into the lamina propria (stage (T1) were aneuploid. Twenty of 24 muscle invasive tumours (stage T2 to T4) were aneuploidy. Unlike the non-invasive tumours (stage $\mathrm{Ta}$ and $\mathrm{T} 1$ ), nonperitetraploid aneuploid lines predominated (14 of 20) in these tumours.

This difference in ploidy between superficial and advanced tumours was highly significant $(p=0.001)$. Furthermore, although the proportion of non-

Table 3 Ploidy in various stages of disease

\begin{tabular}{lcllll}
\hline & & \multicolumn{2}{l}{ Aneuploid } & \\
\cline { 3 - 4 } Stage & Diploid & Peritetraploid & Non-peritetraploid & Total \\
\hline Ta & 18 & 3 & 0 & 21 \\
T1 & 7 & 4 & $5^{*}$ & 16 \\
T2-4 & 4 & 6 & $14 \dagger^{+}$ & 24 \\
Total & 29 & 13 & 19 & 61 \\
\hline
\end{tabular}

*Including one multiploid tumour; tincluding three multiploid tumours.

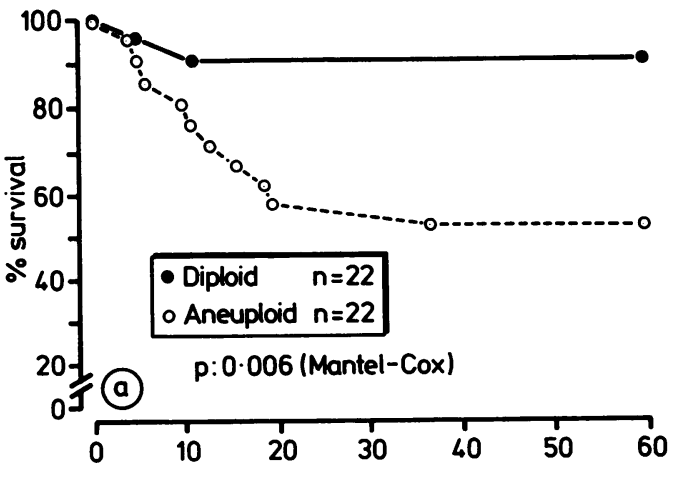

Fig la Kaplan-Meier survival curves for patients with diploid and aneuploid tumours.

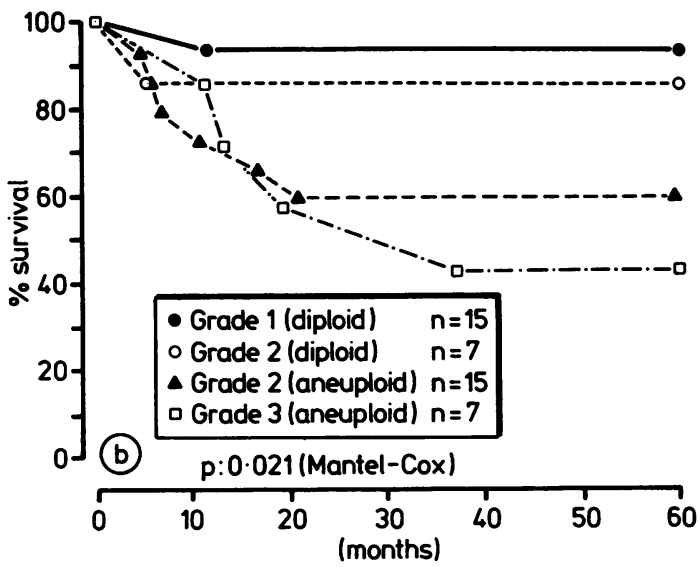

Fig lb Kaplan-Meier survival curves for patients with grade 1 (all diploid), diploid grade 2, aneuploid grade 2, and grade 3 (all aneuploid) tumours. The grade 2 patient group is separated into different prognostic subgroups.

peritetraploid aneuploid lesions among the advanced tumours was considerably larger than among the superficial ones $(70 \%$ compared with $40 \%)$, this tendency was not significant $(p=0 \cdot 11)$. This may have been due to the small number of cases in each subgroup. All multiploid tumours showed advanced disease (stage T2 to T4).

PLOIDY IN RELATION TO FIVE YEAR SUR VIVAL With the exception of the 17 patients who died from causes unrelated to the tumour, those with diploid carcinoma showed a significantly more favourable five year survival than those with aneuploid carcinoma. Only two of the 22 diploid patients $(10 \%)$ died from disease, compared with 11 deaths $(50 \%)$ among the 22 patients with aneuploid cells $(\mathrm{p}=$ 0.006) (fig 1a). 
With regard to five year suvival in relation to histological grade, consideration of ploidy showed a clear demarcation among the patients with grade 2 carcinoma into a diploid subgroup with a more favourable outcome (five year survival of $85 \%$ ), approaching that of grade 1 tumours, and an aneuploid subgroup with a worse prognosis (five year survival of $60 \%$, $p=0.021$ ) (fig 1b). Survival of both these subgroups showed no significant difference from that of the patient groups with grade 1 and grade 3 carcinoma, respectively ( $p=0.54$ and $p=0.43$ ).

The same tendencies were observed in those cases which had been classified with complete agreement in histological grading procedure $(n=34)$. This is illustrated by the five year survivals of the grade $1(n=$ $11)$, the diploid grade $2(n=5)$, the aneuploid grade $2(n=13)$, and the grade $3(n=5)$ tumours, which amounted to $100,80,60$, and $40 \%$, respectively $(p=0.016)$. In addition to clinical staging, determination of ploidy did not contribute to prediction of survival. Almost all patients with superficial carcinoma (stage Ta and T1) (26 of 27), whether diploid or aneuploid, remained alive, and among patients with advanced disease (stage T2 to T4) discrimination between diploid and aneuploid tumours was of little use because all the lesions but two were aneuploid ( 15 of 17).

\section{PLOIDY IN RELATION TO RECURRENCE AND PROGRESSION}

Among the patients with conservatively managed superficial carcinoma (stage $\mathrm{Ta}$ and $\mathrm{T} 1$ ), aneuploidy of the initial tumour was not associated with a higher incidence of recurrence. An equal proportion of patients with diploid (13 of 25 ) and aneuploid (seven of 12) tumours had recurrent disease in the bladder mucosa. In addition, the overall incidence of recurrence, defined as the number of recurrences during months of follow up, did not differ significantly for both groups $(0.040$ and 0.037 recurrences per month, respectively). The prognostic value of ploidy with respect to progressive recurrence was more convincing. Twenty three patients with diploid tumour and seven with aneuploid tumour had no progressive recurrence; one and five, respectively, did have recurrent disease $p=0.004$. Of 36 patients with a conservatively treated superficial primary tumour (stage Ta and T1), 23 patients with diploid tumour and seven with aneuploid tumour had no progressive recurrence; one and five, respectively, did have progressive disease $(p=0.004)$. Of these, six cases, two progressed from low grade (grade 1 or 2) to high grade (grade 3 ) carcinoma without obvious muscle invasion, and in one case this was accompanied by flat carcinoma in situ; four patients presented with recurrent muscle invasion. Two patients died from disseminated car- cinoma and two died shortly after local radiotherapy or cytostatic bladder instillation, without manifest carcinoma, although this was not verified by necropsy. Of the remaining two patients, one stayed free of disease after transurethral resection and the other successfully underwent cystectomy.

\section{Discussion}

This study shows the strong correlation between ploidy and tumour grade and stage. Our findings correspond with reported data obtained from fresh tumour specimens ${ }^{2124}$ and confirm that flow cytometry on routinely processed, paraffin embedded specimens is a relevant method of determining nuclear DNA in urinary bladder carcinoma.

We found a strong association between ploidy and survival and the prognostic importance of ploidy was shown, especially in grade 2 carcinomas. As all grade 1 tumours were diploid and almost all grade 3 carcinomas were aneuploid, ploidy was obviously not an additional predictive factor for both these groups. A striking division, however, was seen among patients with a grade 2 carcinoma, for they could be divided into a diploid subgroup with a clinical outcome approaching that for grade 1 (five year survival of $85 \%$ ) and an aneuploid subgroup with a far less favourable outcome (five year survival of $60 \%$ ). The question can therefore be raised as to whether the diploid grade 2 tumours are biologically different from grade 1 carcinomas. Our results obviously show that grade 2 transitional cell carcinomas constitute a heterogeneous group of tumours. Although previous flow cytometric studies also showed a clear division among grade 2 carcinomas on account of ploidy, ${ }^{2023}$ to our knowledge, no study has shown this heterogeneity by correlating ploidy with clinical outcome.

Evaluation of ploidy in addition to stage of the disease yielded less impressive results. Among the superficial lesions (stage Ta and T1) aneuploidy was associated with invasion of the lamina propria, which might be regarded as a hallmark of aggressive behaviour. Nevertheless, of 36 conservatively treated patients only one died of carcinoma within five years. Ploidy, therefore, did not indicate more aggressive management.

All but four of the 24 patients with advanced carcinoma (stage T2 to T4) displayed aneuploid tracings, indicating that ploidy does not provide prognostic information in addition to tumour stage. High grade, muscle invasive transitional cell carcinoma in many instances develops without a previous history of recurrent disease, ${ }^{27}$ and is treated preferably by radical surgery. On the other hand, patients with superficial papillary carcinoma are principally managed conservatively. Nevertheless, up to $25 \%$ eventually have 
progressive recurrence. ${ }^{78}$ Much attention has been paid to the identification of this group - for example, by immunohistochemistry (presence or loss of carcinoembryonic antigen, $\mathrm{ABO}(\mathrm{H})$ isoantigens, Thomsen Friedenreich antigen)-because early detection of patients at risk would allow more aggressive treatment to be given at an earlier stage of the disease. In our series 36 patients initially presented with a superficial carcinoma (stage $\mathrm{Ta}$ and $\mathrm{T} 1$ ) and six of them eventually progressed to high grade or muscle invasive carcinoma. In five of these cases the primary tumour exhibited aneuploid tracings and moreover, these tumours comprised almost half (five of 12) of all aneuploid cases: determination of ploidy therefore seems to be a valuable and sensitive tool in predicting progressive recurrence. This finding agrees with those of a previous study by Gustafson et al, who measured nuclear DNA by flow cytometric analysis of bladder washing specimens. ${ }^{28}$ Automated flow cytometry provides a rapid and objective predictor of progressive disease, which, unlike the present immunohistochemical techniques, is not hampered by technical failures or subjective interpretation. ${ }^{29} 30$

In conclusion, our results, although based on a relatively small number of patients, show that ploidy is of prognostic importance in addition to histological grade, especially in the heterogeneous group of grade 2 carcinomas, for determining outcome. Our findings suggest that in superficial recurrent bladder carcinoma ploidy of the initial tumour may predict the aggressive behaviour of future recurrences and may thus help to ensure more appropriate management of the disease.

\section{References}

1 Kern WH. The grade and pathological stage of bladder cancer. Cancer 1984;53:1185-9.

2 Bergkvist A, Ljungqvist A, Moberger G. Classification of bladder tumours based on the cellular pattern. Acta Chir Scand 1965;30:371-8.

3 Koss LG. Tumors of the urinary bladder. In: Atlas of tumor pathology. 2nd series, fascicle 11. Armed Forces Institute of Pathology, Washington DC, 1975.

4 World Health Organisation. Histological typing of urinary bladder tumours. International histological classification of tumours. Geneva: WHO, 1973.

5 Busch C, Engberg A, Norlen BJ, Stenkvist B. Malignancy grading of epithelial bladder tumours. Scand $J$ Urol Nephrol 1975;1:143-8.

6 Ooms ECM, Anderson WAD, Alons CL, Boon ME, Veldhuizen RW. Analysis of the performance of pathologists in the grading of bladder tumours. Hum Pathol 1973;14:140-3.

7 Melicow MM. Tumors of the urinary bladder: a clinicopathological analysis of over 2,500 specimens and biopsies. $J$ Urol 1955;74:498-503.

8 Barnes R, Hadley H, Dick A, Johnston O, Dexter J. Changes in grade and stage of recurrent bladder tumours. $J$ Urol 1977;118:177-8.

9 Lamb D. Correlation of chromosome counts with histological appearances and prognosis in transitional cell carcinoma of bladder. Br Med J 1967;1:273-7.
10 Spooner ME, Cooper EH. Chromosome constitution of transitional cell carcinoma of the urinary bladder. Cancer 1972;29:1401-12.

11 Falor WH. Chromosomes in non-invasive papillary carcinoma of the bladder. JAMA 1971;21:791-4.

12 Lederer B, Mikuz G, Guetter W, zur Nedden G. Zytophotometrische Unteršuchungen von Tumoren des Uebergangsepithels der Harnblase. Vergleich zytophotometrischer Untersuchungsergebnisse mit dem histologische Grading. Beitratge zur Pathologie 1972;147:379-89.

13 Fossa SD, Kalhus $O$, Scott-Knudsen $O$. The clinical and histopathological significance of Feulgen DNA values in transitional cell carcinoma of the human urinary bladder. Eur $J$ Cancer 1977;13:1155-62.

14 Wiley EL, Mendelsohn G, Droller M, Eggleston JC. Immunoperoxidase detection of carcinoembryonic antigen and blood group substances in papillary transitional cell carcinoma of the bladder. $J$ Urol 1982;128;276-80.

15 Decenzo JM, Howard P, Irish CE. Antigenetic deletion and prognosis of patients with stage A transitional cell bladder carcinoma. J Urol 1975;114:874.

16 Coon JS, Weinstein RS, Summers JL. Blood group precursor T-antigen expression in human urinary bladder carcinoma. Am J Clin Pathol 1982;77:692-9.

17 Pauli BU, Cohen SM, Alroy J, Weinstein RS. Desmosome ultrastructure and the biological behavior of chemical carcinogen induced urinary bladder carcinomas. Cancer Res 1978;38:3276-85.

18 Caruthers JMS, Bonneville MA. Luminal plasma membrane alternations in bladder cancer. Invest Urol 1980;17:364-72.

19 Collste LS, Devonec M, Darzynkiewicz R et al. Bladder cancer diagnosis by flow cytometry. Correlation between cell samples from biopsy and bladder irrigation fluid. Cancer 1980;45:2389-94.

20 Tribukait B, Gustafson H, Esposti PL. The significance of policy and biological evaluation of bladder tumours: a study of 100 untreated cases. Br J Urol 1982;54:130-5.

21 Farsund T, Hoestmark JG, Laerum OD. Relation between flow cytometric DNA distribution and pathology in human bladder cancer. Cancer 1984;54:1771-7.

22 Chin JL, Huben RP, Nava E et al. Flow cytometric analysis of DNA content in human bladder tumors and irrigation fluids. Cancer 1985;56:1677-81.

23 Gustafson H, Tribukait B. Characterisation of bladder carcinoma by flow DNA analysis. Eur Urol 1985;11:410-17.

24 Wijkstroem H, Gustafson H, Tribukait B. Deoxyribonucleic acid analysis in the evaluation of transitional cell carcinoma before cystectomy. J Urol 1985;132:894-8.

25 Hedley DW, Friedlander ML, Taylor IW, Rugg CA, Musgrove EA. Method for analysis of cellular DNA content of paraffin-embedded pathological material using flow cytometry. J Histochem Cytochem 1983;31:1333-5.

26 Union Internationale contre le Cancer. TNM classification of malignant tumours. Third ed. Geneva: International Union against Cancer, 1978.

27 Kaye KW, Lange PH. Mode of presentation of invasive bladder cancer: reassessment of the problem. $J$ Urol 1982;128:31-3.

28 Gustafson H, Tribukait B, Esposti PL. DNA profile and tumour progression in patients with superficial bladder tumours. Urol Res 1982;10:13-18.

29 Juhl BR, Hartzen SH, Hainau B. A, B. H antigen expression in transitional cell carcinomas of the urinary bladder. Cancer 1986:57:1768-75.

30 Srinivas V, Kiruluta HG. $\mathrm{ABO}(\mathrm{H})$ isoantigens in bladder tumours: a new technique of quantitative analysis. $J$ Urol 1984;131:245-8.

Requests for reprints to: Dr C E M Blomjous, Department of Pathology, Free University Hospital, Amsterdam, The Netherlands. 